\title{
ON THE SOLVABILITY IN LOCAL EXTENSIONS
}

\begin{abstract}
Akram Lbekkouri
Abstract

The aim of this paper is the study of the solvability of finite extensions of local fields in the general case that is when the residue field is not necessarily perfect.
\end{abstract}

\section{Perfectness of residue fields in ramification theory}

In case of a perfect residue field or at least when the residue extension is assumed to be separable (ie. classical case) the ramification theory is well studied for several decades.

However, in case of the residue field is assumed not to be necessarily perfect and the residue extension not to be necessarily separable (ie. general case) one has to redefine the notion of totally (wildly) ramification namely, the filtrations upper and lower of the inertia group. Indeed, ramification theory in this case remains an absolutely mysterious enigma with a lot of unsolved questions in spite of the works of K.Kato, A.Abbes, T.Saito and many others.

By local field, we mean a complete field with respect to a discrete valuation, hence its residue field can be infinite, even imperfect.

Key Words: Inertia group, General case, Imperfect residue field, N-dimensional a local fields.

2010 Mathematics Subject Classification: 11S15.

Received:November, 2012

Accepted:December, 2012. 


\subsection{Sketch on classical ramification}

Let $L / K$ be a finite galois extension of local fields. In this paragraph only, the residue fields are assumed to be perfect or at least the residue extension is assumed to be separable. In this case we can easily define two canonical filtrations $\left(G_{i}\right)$ (the lower ramification groups) and $\left(G^{i}\right)$ (the upper ramification groups) in $G$ that are decreasing sequences of subgroups of $G$. The $i-t h$ lower ramification group is defined to be $G_{i}=\{\sigma \in G ; w(\sigma(\alpha)-\alpha) \geq i+1$ for all $\alpha \in \mathcal{R}_{L}$, where $\mathcal{R}_{L}$ is the ring of the discrete valuation $w$. The numbers $i$ such that $G_{i} \neq G_{i+1}$ are called ramification numbers (or jumps, breaks). The two said filtrations are related by the Hasse-Herbrand functions $\phi$ and $\psi$ used to be calculated in terms of orders of $G_{i}$. Serre in ( [3] $\left.C h: I V\right)$, defined the classical lower subgroups ramification filtration (above) as it is done in [4], then by using the said functions he obtained an upper subgroups ramification filtration $\left(G^{n}\right)_{n \geq 1}$, related to each other by the formula $\left(G_{n}=G^{\phi(n)}\right)_{n \geq 1}$ and $\left(G^{n}=G_{\psi(n)}\right)_{n \geq 1}$ hence the knowing of the $G_{n}$ is equivalent to the last one of the $G^{n}$; nevertheless they behave very differently; the upper one behaves well under quotient subgroups; meanwhile, the lower one behaves well under taking subgroups.

It is worthy to note that the said filtrations are compatible with the subextensions in $L / K$. In the sense that, if $H$ is a normal subgroup of $G$, we can get the filtrations relative to $H$ by taking $H_{i}=G_{i} \cap H$ for any $i-t h$ lower ramification group ([3] $C h: 4 \S .1$ Proposition 2) and $(G / H)^{v}=\left(G^{v} H\right) / H$ for any number $v$ ([3] $C h: 4 \S .3$ Proposition 14). Furthermore, if $L / K$ is abelian when the residue field is quasi-finite, we have that the "upper" filtration of $G$ is compatible with the reciprocity map $\theta: K^{\star} \rightarrow G$ in the sense that $\theta\left(U_{i}\right)=G^{i}$ for all $i=0,1, \ldots, n$, and $\left(U_{i}\right)$ is the filtration of the units group in $K^{\star}$. The compatibility of the "upper" filtration of $G$ with the class field theory is studied in detail in ( [3] $C h: X V)$.

Consequently, in classical case the solvability of $G_{0}$ the inertia subgroup of $G$ can be easily proved ( [3] $\S .2 C h: I V$ page:68 Corollary: 5 ), since the quotient of any two successive lower ramification groups is abelian. In fact it is either cyclic or a direct sum of groups of order $p$. Meanwhile, the solvability of $G$ itself requires the finiteness of the residue field, see Counter-example (1.1) that follows.

Example 1.1. (Counter-example)

Consider any finite galois and non solvable extension $L / \mathbb{Q}$ whose galois group is $\operatorname{gal}(L / \mathbb{Q})$ of course non solvable, which acts on a Laurent series in $L((t))$ by acting on coefficients. Then we use $L((t)) / \mathbb{Q}(t))$ as a galois extension of local fields. Its galois group is $\operatorname{gal}(L / \mathbb{Q})$ too and its inertia group is trivial. 


\subsection{General case (Imperfect residue field)}

First note that in the general case, a theory of upper numbering ramification subgroups corresponding to a theory of the "lower" ramification subgroups by use of some means like the Hasse-Herbrand $\varphi, \psi$ functions does not exist, till now.

All that has been done is a "lower" ramification theory which is obtained from the double-filtration given in ([4]) see paragraph $\S .2 .1$ that comes. Meanwhile, the ramification filtration in the group $G$ does not determine that in $H$. Indeed, for $L / K$ a finite galois extension of local fields in the general case, $\mathcal{R}_{L}$ the discrete valuation ring of $L$ may not be generated by only one element over $\mathcal{R}_{K}$ (ie. not necessarily monogenic), when we are in the inseparable case.

Example 1.2. Take the rings $R_{1}=\mathbb{F}_{p}\left[X^{p}, Y^{p}\right]$ and $R_{2}=\mathbb{F}_{p}[X, Y]$ with $X$ and $Y$ transcendant over $\mathbb{F}_{p}$. So, $k=\mathbb{F}_{p}\left(X^{p}, Y^{p}\right)$ and $l=\mathbb{F}_{p}(X, Y)$ define an inseparable extension of fields $\bar{L} / \bar{K}$ in characteristic $p$. It satisfies the property that the ring $R_{2}$ cannot be generated over $R_{1}$ by only one element. Then consider the rings of Witt vectors over $R_{1}$ and $R_{2}$ say $W R\left(R_{1}\right)$ and $W R\left(R_{2}\right)$. $\bar{L} / \bar{K}$ is the residue extension of the fraction fields $L / K$ of the corresponding rings of Witt vectors. Therefore, $W R\left(R_{2}\right)$ cannot be generated by only one element over $W R\left(R_{1}\right)$, although $L / K$ is a finite galois extension.

Moreover, recently for a theory of the upper numbering filtration, Abbes and Saito made a different interpretation that can be generalized in the general situation, giving rise to a quite well-behaved upper-number filtration. To sum up, they define two decreasing filtrations by ramification groups on the absolute galois group such that in the classical case where the residue field is perfect, we recover the classical upper numbering filtration. The definition uses rigid geometry and log-structures. See [1].

\section{The question of solvability}

Let $L / K$ be a finite galois extension of any local fields. Write $G=\operatorname{gal}(L / K)$ for its galois group, $\mathcal{R}_{L}$ the discrete valuation ring and $\mathcal{M}_{L}$ its maximal ideal. Its degree splits such a way $[L: K]=e f=e_{\text {tame }} e_{\text {wild }} f_{\text {sep }} f_{\text {insep }},\left(e_{\text {wild }}\right.$ and $f_{\text {insep }}$ must be a power of $p, e_{\text {tame }}$ is prime to $p$, meanwhile $f_{\text {sep }}$ need not be necessarily prime to $p$ ).

Note that its residue extension $\bar{L} / \bar{K}$ is normal, see for example ([3] $C h: 1$ $\S .7$ Proposition.20), but need not be separable. Furthermore, consider $D$ the set of all automorphisms of $\bar{L}$ unvarying all elements of $\bar{K}$ there is a natural surjective homomorphism from $\varphi: G \rightarrow D$. Indeed, let $g \in G, g$ preserves $\mathcal{R}_{L}$ as well as $\mathcal{M}_{L}$. Therefore $g$ induces an automorphism of $\bar{L}=\mathcal{R}_{L} / \mathcal{M}_{L}$. Since 
$g$ fixes each element of $K$ it fixes each element of $\bar{K}$ as well, the surjectivity of $\varphi$ is proved in the Proposition referred above.

\subsection{A filtration of the inertia group}

Let $L / K$ be any finite galois extension of local fields (complete with discrete valuation), with the respective rings of integers $\mathcal{R}_{K} \subset \mathcal{R}_{L}$, and the prime ideals $\mathcal{M}_{K} \subset \mathcal{M}_{L}$ and residue fields $\bar{K} \subset \bar{L}, G=\operatorname{gal}(L / K)$ being the galois group. The inertia group $G_{0}$ is given by $G_{0}=\left\{\sigma \in G, \forall x \in \mathcal{R}_{L} ;(x-\sigma(x)) \in \mathcal{M}_{L}\right\}$. Here we give some outline of Zariski-Samuel ramification filtration. Anyway, by use of a filtration coming from it we prove Proposition (2.1), that follows. In ( [4]ch:V §.10 page 294) following Hilbert's way in the definition of ramification subgroups of the galois group, Zariski and Samuel define their filtration as follows.

For any positive integer $n \geq 1$, they define the $n$-th ramification group $G_{n}$ as the subset of $G$ consisting of all automorphisms $\sigma \in G$ such that $\sigma(x) \equiv$ $x$ modulo $\mathcal{M}_{L}^{n+1}$ for every $x \in \mathcal{R}_{L} . G_{n}$ is then the kernel of the action on $\mathcal{R}_{L} / \mathcal{M}_{L}^{n}$. They establish that $G_{n}$ are invariant subgroups of $G$, and the quotients $G_{n} / G_{n+1}$ are abelian for $n \geq 1$ see ( Lemma 1. page 295). Meanwhile, $G_{0} / G_{1}$ need not be abelian in general case, see ( [4] page 297 Last line). Indeed, there are extensions where $f_{\text {insep }}>1$ and $e>1$ but for which there does not exist a subgroup which can "separate" ferocious from wild ramification. So a second filtration $H_{n}$ becomes necessary. To sum up, in the general case the ramification filtration is a double-filtration.

By use of the homomorphism,

$$
\begin{array}{ll}
\lambda: \quad & G_{0} \rightarrow \bar{L}^{\star} \\
& \sigma \mapsto \lambda(\sigma)=\overline{(\sigma(\pi) / \pi)}=u_{\sigma}
\end{array}
$$

$H_{1}$ is defined as its kernel that is the subgroup of all automorphisms $\sigma$ in $G_{0}$ such that $u_{\sigma} \equiv 1$ modulo $\mathcal{M}_{L}$; that is such that $\sigma(\pi)-\pi \in \mathcal{M}_{L}^{2}$.

Likewise, $H_{i}$ is defined to be the kernel of the homomorphism;

$$
\begin{aligned}
\lambda_{i}: & G_{i} \rightarrow(\bar{L},+) \\
& \sigma \mapsto \lambda_{i}(\sigma)=y_{\sigma}
\end{aligned}
$$

that is the subgroup of all automorphisms $\sigma$ in $G_{i}$ such that $y_{\sigma} \equiv 0$ modulo $\mathcal{M}_{L}$, where $y_{\sigma}$ is the integer $y_{\sigma} \in \mathcal{R}_{L}$ satisfying $\sigma(\pi)-\pi=y_{\sigma} \pi^{i}$, that is such that $\sigma(\pi)-\pi \in \mathcal{N}_{L}^{i}$.

It is clear that $G_{i} \supseteq H_{i}$ for every $i \geq 1$ the equality occurs when the residue fields extension is separable. See ( [4] Ch: V $\S .10$ page 296 ).

That is $\sigma \in H_{i}$ implies that $\sigma(x) \equiv x$ modulo $\mathcal{M}_{L}^{i+1}$ for every $x \in \mathcal{R}_{L} . H_{i}$ is then the kernel of the action on $\mathcal{M}_{L} / \mathcal{M}_{L}^{i}$ for $i \geq 1$.

Applying this filtration for the particular case when the residue extension $\bar{L} / \bar{K}$ is separable (ie. the classical case), we have $\left(H_{n}=G_{n}\right)_{n \geq 1}$. 


\subsection{Results}

Proposition 2.1. Let $L / K$ be a finite galois extension of local fields with imperfect residue class fields. Denote by $G_{0}$ the inertia group of $\operatorname{gal}(L / K)$. Then $G_{0}$ is solvable, furthermore it is cyclic in characteristic zero case.

Proof. A uniformizer $\pi$ of $L$ being fixed and, let us fix a set of generators of the residue field extension and their lifts $u_{1}, \ldots, u_{n}$ to $\mathcal{R}_{L}$. Put it another way, $\mathcal{R}_{L}$ is generated by $\pi, u_{1}, \ldots, u_{n}$ as an $\mathcal{R}_{K}$-algebra, where $\pi$ has valuation 1 , and $u_{i}$ are units.

Consider the map:

$G_{0} \rightarrow \bar{K}^{\star}$

$g \mapsto \overline{g(\pi) / \pi}$

it is clear that this is a homomorphism, write $J_{1}$ for the kernel of this map, it is too clear that $J_{1}=H_{1}$ in Zariski-Samuel notation. Then again consider the map:

$J_{1} \rightarrow \bar{K} \oplus \bar{K} \oplus \ldots \oplus \bar{K} ;(n+1$ of them $)$

$g \mapsto \overline{(g(\pi)-\pi) / \pi^{2}}, \overline{\left(g\left(u_{1}\right)-u_{1}\right) / \pi}, \ldots, \overline{\left.\left(g\left(u_{n}\right)-u_{n}\right) / \pi\right)}$ where $\overline{(g(\alpha)-\alpha) / \pi^{i}}$ is the residue class of $(g(\alpha)-\alpha) / \pi^{i} \bmod \pi$, that is also a homomorphism, and $J_{2}$ is the kernel. Then we continue to consider:

$J_{2} \rightarrow \bar{K} \oplus \bar{K} \oplus \ldots \oplus \bar{K} ;(n+1$ of them $)$

$g \mapsto\left(\overline{(g(\pi)-\pi) / \pi^{3}}, \overline{\left(g\left(u_{1}\right)-u_{1}\right) / \pi^{2}}, \ldots, \overline{\left.\left(g\left(u_{n}\right)-u_{n}\right) / \pi^{2}\right)}\right.$,

and so on and so forth, till we get a trivial $J_{r}$. Of course this filtration stabilizes, since $\mathcal{R}_{L} \simeq \lim \mathcal{R}_{L} / \mathcal{M}_{L}^{i}$

From this, in case of the residue fields have characteristic $p>0$, it is clear that $J_{1}$ has a filtration by normal subgroups $J_{i}$ where the subquotients $J_{i} / J_{i+1}$ are $p$-elementary abelian groups as $J_{i} / J_{i+1}$ injectively maps to $\left(1+\mathcal{M}_{L}^{i}\right) /\left(1+\mathcal{M}_{L}^{i+1}\right)$ which is canonically isomorphic to $(\bar{L},+)$ for $i \geq 1$.

Furthermore, $G_{0} / J_{1}$ is cyclic as it injectively maps to $\mathcal{R}_{L}^{\star} /\left(1+\mathcal{M}_{L}\right) \simeq\left(\bar{L}^{\star}, \times\right)$, as well as to $A u t_{\bar{L}}\left(\mathcal{M}_{L} / \mathcal{M}_{L}^{2}\right) \simeq\left(\bar{L}^{\star}, \times\right)$ too, and the field $\bar{L}$ is of characteristic $p$ (remark the order of $G_{0} / T_{1}$ is prime to $p$ if $p \geq 3$ ). Worthy to note that the maximal tamely ramified subfield $\mathrm{T}$ of $\mathrm{L}$ corresponds to the group $G_{T}=$ $G_{0} \cap J_{1}=J_{1}$. Finally, $J_{1}$ is a $p$-group of order $e_{\text {wild }} f_{\text {insep }}$, therefore its solvability implies the last one of $G_{0}$.

In case of the residue fields are of characteristic zero, since for $i \geq 1$ the subquotients $J_{i} / J_{i+1}$ are isomorphic to a subgroup of $(\bar{L},+)$, considered as an additive group. $(\bar{L},+)$ having no finite subgroup except $\{0\}$ therefore the $J_{i}$ are trivial for all $i \geq 1$ in consequence $G_{0}$ is cyclic. Which ends the proof.

The result of the solvability of the inertia group can be deduced from the work of I.B.Zhukov in [2] but not for the general case. Indeed, he added the strong hypothesis that $\left[\bar{K}: \bar{K}^{p}\right]=p$ where $k$ is the residue field, that this $K$ is 
particularly assumed to be a two-dimensional local field.

Remark 2.2. It is well known, since Hilbert's time, that $G / G_{0}$ has order $f_{\text {sep }}$, where $G_{0}$ is the inertia group. Furthermore, one can always find a normal subgroup $G_{1}$ of $G_{0}$ of order $e_{\text {wild }} f_{\text {insep }}$. Meanwhile the existence of a normal subgroup of $G_{1}$ of order $e_{\text {wild }}$ or $f_{\text {insep }}$ is not assured. By other words, the wild ramification and ferociously ramification need not be necessarily, "separated" in two different extensions.

Proposition 2.3. Let $K$ be a local field, and let $L / K$ be a finite galois extension. Then $L / K$ is solvable if and only if the maximal separable subextension of $\bar{L} / \bar{K}$ is solvable.

Proof. Consider $G=\operatorname{gal}(L / K)$, and $G_{0}$ its inertia group. By use of the current notations the isomorphism induced by the surjective homomorphism $\varphi$ defined above, makes $G / G_{0}$ isomorphic to the galois group of the maximal separable subextension of $\bar{L} / \bar{K}$ which equals $D$. From (2.1) $G_{0}$ is solvable, the well known result of classical group theory " $G$ is solvable if and only if $G / G_{0}$ and $G_{0}$ both of them are solvable" ends the proof.

Remark 2.4. Consequently, in the classical case that is when the residue extension is assumed to be separable (particularly in case of perfect residue fields) we have that the galois group of $L / K$ is solvable if and only if the galois group of $\bar{L} / \bar{K}$ is solvable.

Furthermore, it is worthy to mention, the following result:

A field is called to be "1-dimensional " if it is complete with respect to its discrete valuation and has a finite residue field, it is called to be "2-dimensional " if it is complete with respect to its discrete valuation and has a residue field which is itself "1-dimensional ", and so on we can define an "n-dimensional " local field. Note that some authors require for the "1-dimensional " local field to have only a perfect residue field. For our next corollary we require necessarily its finitude.

Making use of Proposition (2.3) and since every finite extension of a finite field is cyclic, by induction we can immediately deduce:

Corollary 2.5. Every finite galois extension of a " $n$-dimensional "local field such that the residue field of the corresponding "1-dimensional " field is finite, has necessarily a solvable galois group.

To sum up we get a generalization of Serre's result in ( [3]Ch.IV $\S .2$ Corollary 5). 


\section{References}

[1] A. AbBes And T. SAito, Ramification of local fields with imperfect residue fields, Amer. J. Math. 124 (5) (2002), 879-920.

[2] I.B.ZHUKOV, On ramification theory in the imperfect residue field case, Prepint No. 98-02, Nottingham Univ.,1998. Proceedings of the conference: Ramification Theory of Arithmetic Schemes (Luminy,1999)(ed.B.Erez) accessible on http://family239.narod.ru/math/publ.htm.

[3] J.P.SERRE, Local fields, Springer 1979.

[4] O.ZARISKI AND P.SAMUEL, Commutative algebra, vol.I, Van Nostrand, Princeton, New Jersey, 1958 [Reprinted Springer-Verlag GTM 28 New York, 1960 ].

Akram LBEKKOURI,

BP: 10507

Casa-Bandoeng,

20002 Casablanca, Morocco.

Email: lbeka11@gmail.com 
